\title{
Robotized Inspection System of the External Aircraft Fuselage based on Ultrasound
}

\author{
José Sánz, Manuel Ferre, IEEE, Alvaro Espada, Matias Collar Narocki, José Fernández Pardo
}

\begin{abstract}
This paper describes an automated system that inspects the external surface of an aircraft section. To test the system developed, a part similar to airplane section 19 has been used. The inspection system is based on a robot carrying a headset made up of ultrasound sensors. Data are processed by an ultrasound processing program which maps the mechanical features of the inspected surface. The main requirement of the inspection is to perform the proper contact between the ultrasound sensors and the inspected surface. In order to guarantee suitable contact between them, a robot trajectory correction system running in real time has been applied. It is based on the measures given by four optical sensors located on the sides of the ultrasound headset. The integration and synchronization of the devices mentioned above make it possible to obtain an adequate inspection system for the external aircraft fuselage. Furthermore, inspection time has been signilicantly reduced; at the same time a fully-automated system has been developed to inspect the whole aircraft section surface.
\end{abstract}

\section{INTRODUCTION}

$\mathrm{I}_{\mathrm{is}}$ $\mathrm{N}$ the aerospace industry, one of the most important issues is assuring the cerrification of an aircraft. Nowadays the authorities require that the quality of every part or component be approved as "ready to fly". This reqnirement makes it necessary to inspect every part and component in an aero slnucture, in order to detect potential defects. The inspection of the Carbon Fiber Reinforced Plastic (CFRP) parts represents an important process within the manufacturing flow line. In this context "important" refers not only to quality, but also to cost efficiency within the manufacturing process as a whole.

Considering the design and stress reqnirements of the parts, inspection methods based on ultrasonic technology have been applied to inspect carbon fiber parts. Phase-array pulse-echo has been identified as the ideal technique for the new development of composite laminate parts, because such technique is able to show the internal quality of parts at the required level of resolution and sensitivity [1].

José I. Sánz is with Airbus aeronautical company (e-mail: Jose.Sanz(àairbus.com)

Mannel Ferre is with the Universidad Politécnica de Madrid (corresponding author, phone: +34913363061 ; fax: +3491 336 3010: email: m.fere( $(\hat{\omega}$ upm.es)

Alvaro Espada is with Airbus aeronautical company (e-mail: Alavro.Espada(a) aistus com).

Matias Collar is with the Universidad Politécnica de Madrid (e-mail: mcollar(@etsii.upm.es).

José Femández-Pardo is with the Universidad Politécnica de Madrid (email: jsferpar(àetsii.upn.es)
Regarding inspection of composite materials, typical defects are the following: porosity (voids in the material), delaminating and debondings on the interface line of adhesive. Some of these flaws are due to the manufacturing process itself while others are caused by impacts made to the part for different reasons. This kind of defect needs to be detected and the data postprocessed for subsequent analysis by skilled engineers, in order to give a final order approving safety performances for the working conditions of the aircraft to the authorities.

It is very imporlant to point out that the component must be inspected one hundred percent. ensuring that there are zero areas of non-allowable defects, before it is assembled into the plane. Specifically for the full composite fuselage barrels. the complexity of the geometry makes it necessary to have an extemal system to inspect the part using a contact probe. This is the aim of this study. It is thanks to this development that the technology is now ready to move from 'days' to 'hours' in terms of lead time, representing a big step forward in production processes.

The aerospace indnstry is raising its prodnction rates and moving from mannal mannfacturing by highly-skilled workers to more repetitive processes using automated machines. High productivity implies the need for many inspection facilities able to perform the process or the need to improve and antomate the operation itself. The first option does not fit with increased flexibility in production and. furthermore. such non-recuring expenses make the costs very high. So, the only solntion is to optimize the time reqnired to inspect a component. Comparing the aerospace and automotive industry, many of the automated. lean production concepts and industrial factories developed by car mannfactnrers can be seen as an opporlnnity by aeronautics to make progress in process efficiency. However, the aerospace industry is moving toward a higher level of automatism not only due to the higher production rates to be achieved bnt also dne to the complexity and dimension of the parts being mannfactnred. Composite materials and processes are being developed every year, giving rise to more and more highly integrated strictures. Examples of the new developments can be fonnd in $[2,3]$.

The contents of this article are organized as follows. The cell used for the aircraft section inspection and the corresponding hardware architecture is introduced in section II. Section III describes the process used to obtain the robot trajectories for the external snrface of aircraft sections. 
Section IV focuses on the real-time trajectory correction applied in order to guarantee proper contact between the sensor and the section surface. The next section presents the inspection performance and the main technical results, and also some examples of the records obtained from the surface inspected are explained. Finally, conclusions of this work are discussed in the last section.

\section{INSPECTION SYSTEM STRUCTURE}

The objective of the inspection system developed is to map a 3-D model of the inspected part. In this mapping each point recorded corresponds to ultrasound data obtained by the system at the robot end effector. The steps taken are shown in Figure 1. The starting data are based on the CAD model used to generate the part, similar to the dimensions of the part to be inspected. This CAD model is used to generate the trajectories that the robot has to follow to inspect the part, known as the route file. The next section describes additional details used to generate the routes.

The route file is used by the task controller to decide which part is to be inspected. The user can change the inspection task order or ask to repeat trajectories. These commands are received by the inspection controller in charge of generating the movement orders for the robot. The robot controller manages the coordination of the robot's movement. Robot trajectories are modified in real time according to the information received by the optical sensors located on the robot end effector. The objective is to maintain the proper distance and orientation between the ultrasonic sensors and the part's surface during the inspection data recording process. The Inspection File Generator module is in charge of correctly integrating the information given by the ultrasonic sensors in the positions provided by the inspection controller.

\section{INSPECTION CELL DESCRIPTION}

The inspection cell is made up of a 6-degree of freedom Kuka robot (model KR-150), a Kuka trackmotion along which the robot base moves, and a holder upon which the part to be inspected is mounted. Thanks to the rotatory degree of freedom of the holder, the part can rotate on its axis. Thus, a total of 8 degrees of freedom allow for the full inspection of the corresponding part. The eight degrees of freedom are directly controlled by the robot controller, called KRC [4]. Figure 2 shows a general view of the inspection cell. It shows the robot next to the mounted part, with the appropriate end effector, upon the external rotatory axis.

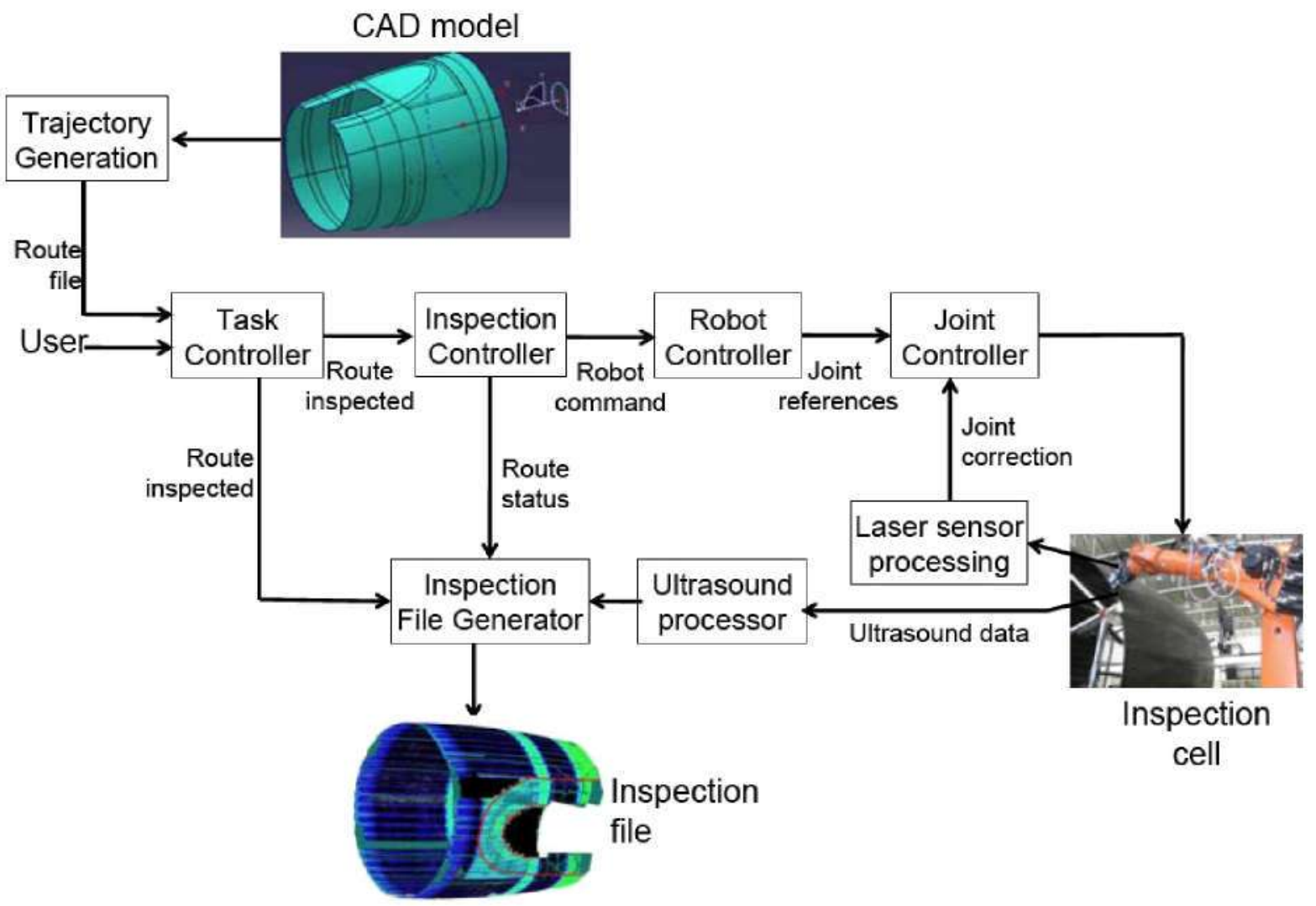

Fig. 1. Diagram of the inspection system. The CAD model is used to calculate the robot trajectories. These trajectories are corrected according to a laser sensor in order to guarantee the contact between ultrasound sensor and the inspected surface. Finally, inspection data are used to reconstruct the inspected part. 


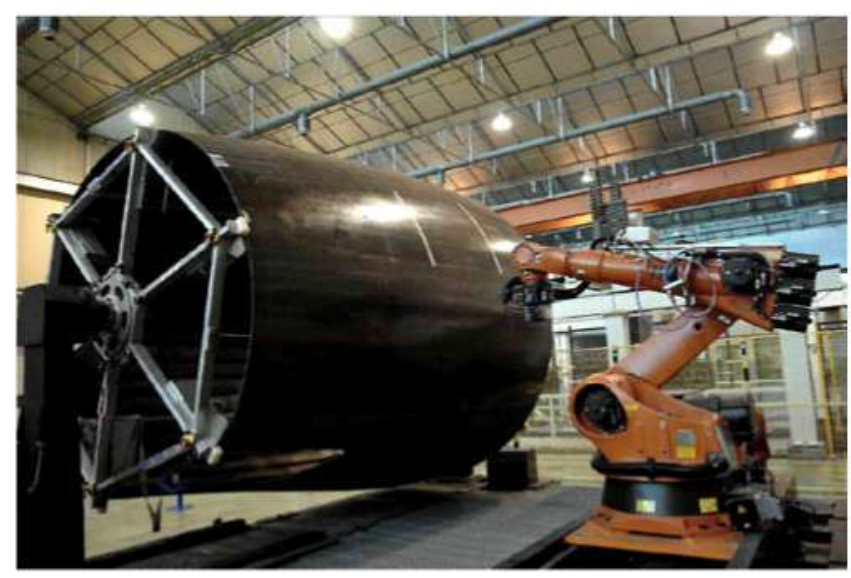

Fig. 2. General view of the cell inspection system.

The robot carries at its end an inspection tool made up of a wheel with an ultrasound system in it. It is used to emit ultrasound and receive the corresponding echoes back. The inspection end effector consists of further elements used during the inspection process like four opticalal sensors and water diffusers for the correct coupling of the ultrasound signal. Figure 3 shows the inspection tool used and the optical sensors.

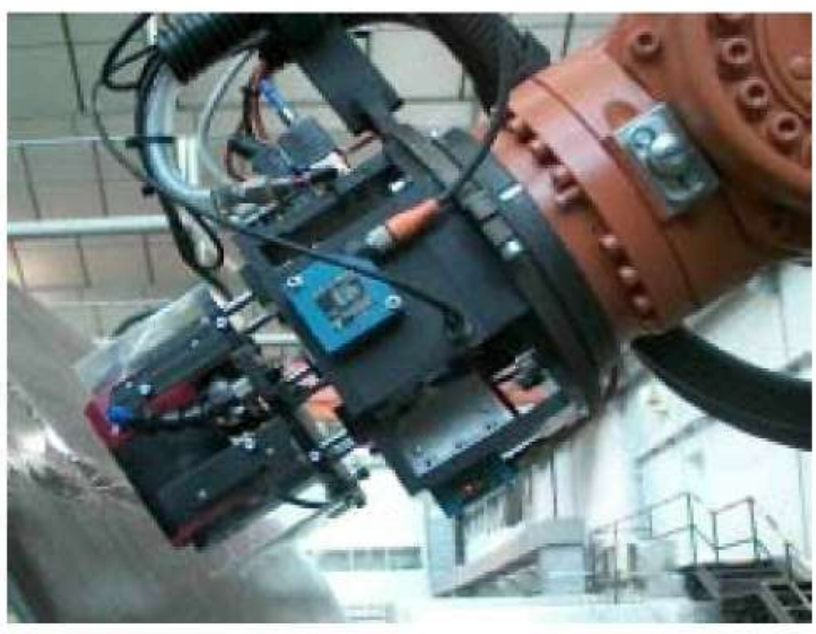

Fig.3. Inspection sensor place at the end robot effector. Four laser are used to measure the distance between end robot and the surface inspected

\section{ROBOT TRAJECTORY GENERATION}

The inspection requires scanning the external surface with an ultrasonic sensor. For this reason, a set of trajectories has been defined so that the robot can guarantee inspection of the whole part. The trajectory calculation has to take into account that the optical sensor system placed in the robot end effector corrects the robot trajectory in real time. The next section describes how it works. Works are performed on a full barrel; which is very similar to an airplane section 19.

In order to calibrate the initial position of the part, the robot has to be moved with a calibration tool to a zero mark of the part. Once the calibration tool is on the mark, the initial position is set in the robot program in order to match the $\mathrm{CAD}$ data with the real part to inspect.

The main requirements to be taken into account when generating trajectories are the following:

- A sample must be taken approximately every $1 \mathrm{~mm}^{2}$ of the part's surface. This factor determines the distance between the ultrasonic sensors and also robot speed, since the speed of movement has to be adapted to the ultrasound captures,

- The axis of the ultrasonic sensors should be perpendicular to the inspection surface, with a tolerance degree of nearly $1^{\circ}$.
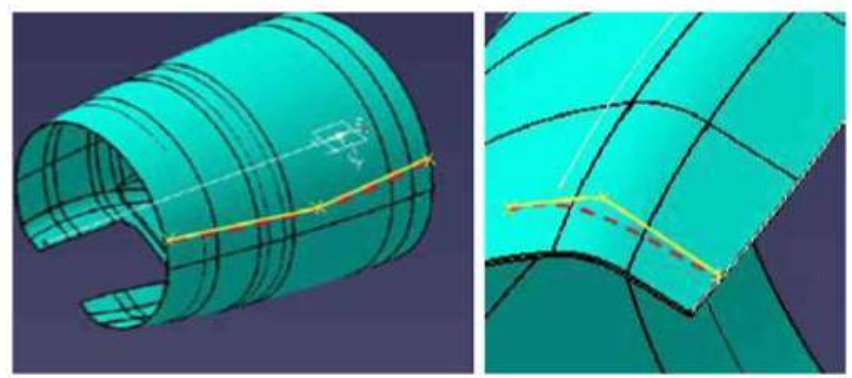

Fig. 4. Defined trajectories for the convex region (left) and for the HTP region (right)

To comply with the second requirement it is necessary to correct the robot trajectory in real time, since the 3-D model may contain uncertainties. The position errors of the inspection end effector must be corrected. This is achieved through the optical sensor system. Robot trajectory calculation takes place offline based on the CAD model of the inspected part. Robot trajectories are defined by a start point, an end point and a number of intermediate points, depending on the trajectory length and curvature. The aim of the intermediate points is to avoid collisions between the end effector and the inspected part in case the trajectory correction system fails. For this reason, the intermediate points locate the end effector some centimeters away from the part. The trajectory correction system is in charge of placing it on the surface. The main restriction for the trajectory calculation is the ultrasonic sensor orientation, which should be perpendicular to the part surface. The correct data capture requires an orientation deviation of less than one degree in relation to the part surface. Further requirements must also be met by the trajectories, such as a minimum overlapping of $5 \%$ between adjacent trajectories.

The section to be inspected shows two clearly defined regions. The first region is the convex region with an almost constant curvature. The second region shows a curvature change, where an inflection area from a convex to a concave external surface can be observed. This region hosts the aircraft Horizontal Tail Plane (HTP). The best inspection 
results have been obtained by using longitudinal trajectories along the part's axis in the constant curvature region; whereas in the region where curvature changes, inspection is optimized following the line of maximum slope.

Robot inspection trajectories in the convex region are drawn by intersecting the part with planes through the part's longitudinal axis (the aircraft axis in aeronautics). These intersections result in a line from which the trajectory start and end points are obtained. Middle points are calculated from the part tangents on the start and end points. In the convex region, tangent intersection provides a unique middle point and guarantees that the tangent intersection is outside the part in order to avoid collisions. Therefore, two rectilinear segments in the convex region define a robot trajectory. Figure 4. left shows a trajectory graph. The whole part inspection requires that the previous process be repeated until $360^{\circ}$ are covered on the angle between the plane and the horizontal. The angle increase between two consecutive planes is given by the effective inspection width of the headset (W), the maximum distance (D) between the inspection trajectory and its projection on the part's axis, and the maximum radio of the part (a), as shown in the following equation:

$$
\Delta \theta=\tan ^{-1}\left(\frac{W}{D}\right)
$$

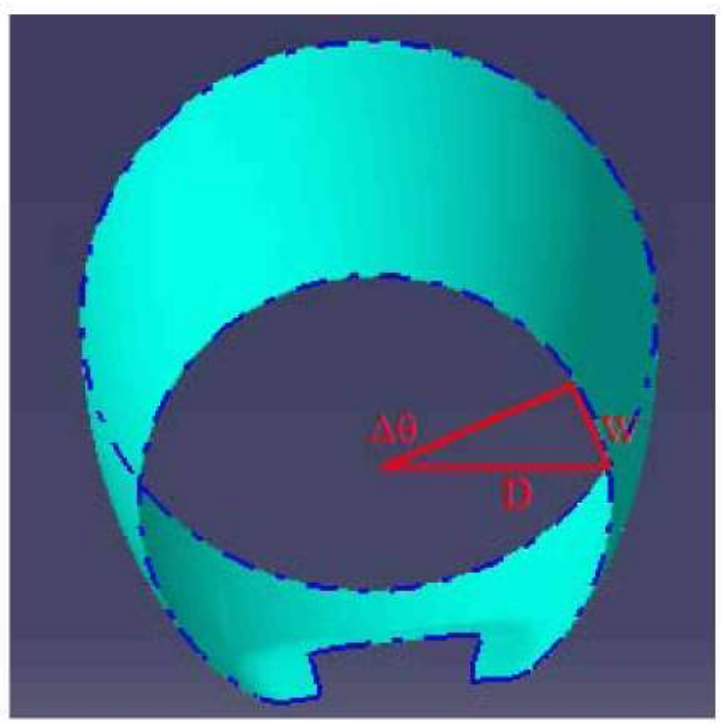

Fig. 5. Angle increase between planes.

Trajectories in the HTP region are calculated from the border region. At each border point, the line of maximum slope is found, which defines the robot trajectory along with the previous point. The maximum and minimum of the robot trajectory are used to define the middle points; usually two middle points are used. A trajectory is calculated based upon displacements along the border, considering the effective width of the inspection ultrasonic system.
Taking into account that the border line is the longest in the double curvature region, obtaining trajectories from this line guarantees the full scanning of this region of the part. These trajectories are radial and meet on the concave region of the part. Figure 4.right shows a trajectory for the double curvature area.

\section{REAL TIME TRAJECTORY CONTROL BASED ON LASER SENSORS}

Potential differences between the CAD-model theoretical part and the real part make it necessary to implement a correction system for the trajectory in real time. The system used must guarantee the proper distance and orientation of

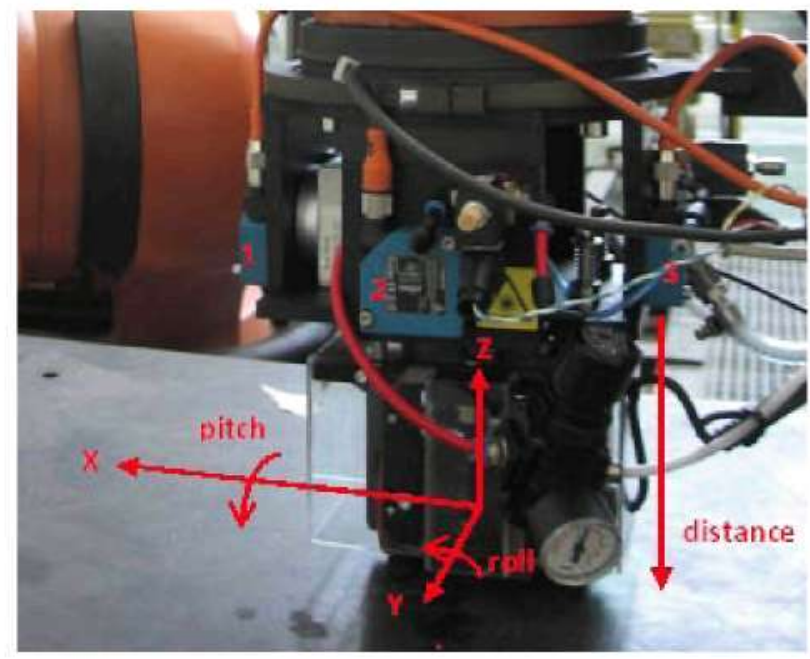

Fig. 6. End effector with laser sensors and directions where the trajectory corrections are applied.

the ultrasonic system while it collects data during the inspection. The most critical aspect is the sensor orientation. The ultrasonic sensor axis should be normal with regard to the inspection surface with a tolerance of $1^{\circ}$.

TABLE I

ROBOT TRAJECTORY CORRECTION FUNCTIONS.

\begin{tabular}{cc}
\hline \hline Correction & \multicolumn{1}{c}{ Analog Input } \\
\hline Distance Z & (Sensor 3 + Sensor 1) / 2 \\
Pitch in X & (Sensor 2 - Sensor 4)+ offset \\
Roll in Y & (Sensor 1 - Sensor 3)+ offset
\end{tabular}

The solution designed is based on the use of 4 laser sensors located on the end effector close to the ultrasonic sensors. The information provided by the laser is processed in order to correct the robot end effector. Commands related to the robot trajectory correction are executed by the Kuka Function Generator [5]. Figure 6 shows how the sensors are located over the end effector, and Table 1 shows how 
correction functions are carried out, where the offset variable is the measure of the sensors when the headset is touching the surface. Other technologies based on the information provided by force sensors [6] or video cameras [7] can be also used for the robot trajectory correction. The main advantages of the laser used in this application are the simplicity of signal processing and the fast sensor response.

As shown in Table 1, laser sensor information is used to control movements on $\mathrm{Z}$ and turns on $\mathrm{X}$ and $\mathrm{Y}$. Movement along $\mathrm{Z}$ shows the end effector proximity to or separation from the part. This movement guarantees that the ultrasonic sensor is in touch with the part and that it does not exert too much pressure on it. Correction takes place according to information provided by sensors 1 to 4 , which are located on the device ends with the ultrasonic. The turn on $\mathrm{X}$ shows the robot wrist roll which uses sensors 1 and 3 . The turn on $Y$ means the robot wrist pitch; its purpose is to guarantee perpendicularity between the header axis and the inspection surface. Sensors 2 and 4, located at the front and at the back of the ultrasonic system, control this movement.

Correction functions are executed by the corresponding functions provided by Kuka to implement controllers. They implement a proportionate controller with a variable gain including dead area and saturation. To obtain the correct values of the gains and saturation limits a number of tests must be carried out in order to adjust the parameters according to the geometry of the part to be inspected. These functions have proven to be sufficient for inspection requirements.

\section{SYSTEM PERFORMANCE}

The developed system has been used to inspect a full barrel similar to an airplane section 19. Its dimensions are approximately $4.0 \mathrm{~m}$. long, with the radius being a maximum of $1.9 \mathrm{~m}$ and a minimum $1.4 \mathrm{~m}$. The generated inspection file has a great deal of data with an ultrasound echo for each $\mathrm{mm}^{\wedge} 2$ and the corresponding spatial position on the inspected part. The ultrasonic sensor is based on a phasearray with 64 lined-up elements that are activated into groups of eight elements. The width of the headset used is $45.6 \mathrm{~mm}$. The effective scanned band width is reduced by $12 \%$, which makes it $40.0 \mathrm{~mm}$., so that band overlapping is assured. If part dimensions and effective band width are considered, then 300 routes $\left(2 * \pi^{*} 1900 / 40\right)$ must be performed in order to scan the entire convex region of the inspected part. Angular distance between routes on the convex area results in $1.2^{\circ}\left(360^{\circ} / 300\right)$. A minimum of $12 \%$ route overlapping is guaranteed in the widest area, while route overlapping in the narrow areas is as high as $34 \%$.

An essential inspection factor is the speed at which the robot drives inspection scanning. This speed affects the quality of the ultrasound data, as well as the trajectory correction in real time. A slow speed guarantees a good capture and trajectory correction, but it will considerably increase inspection time spent on the part, which will worsen the performance of the inspection system. It is necessary to carry out the inspection at the maximum speed at which quality of ultrasound data and trajectory correction are not threatened. After a variety of tests at different speeds, it has been concluded that the ideal inspection speed is $250 \mathrm{~mm} / \mathrm{s}$. At this rate, the total inspection time of the aerodinamical surface of the part with the forementioned dimensions is around 3 hours. A great improvement considering that nowadays manual inpsection takes between 8 and 12 hours.

As a result of a part inspection, a C-Scan file is generated; which is used to develop the pictures shown in Figure 7. This file is processed to analyse possible part errors/defects/flows. If the part is free of flaws, it is issued. If this is not the case, a detailed study of the area should follow.
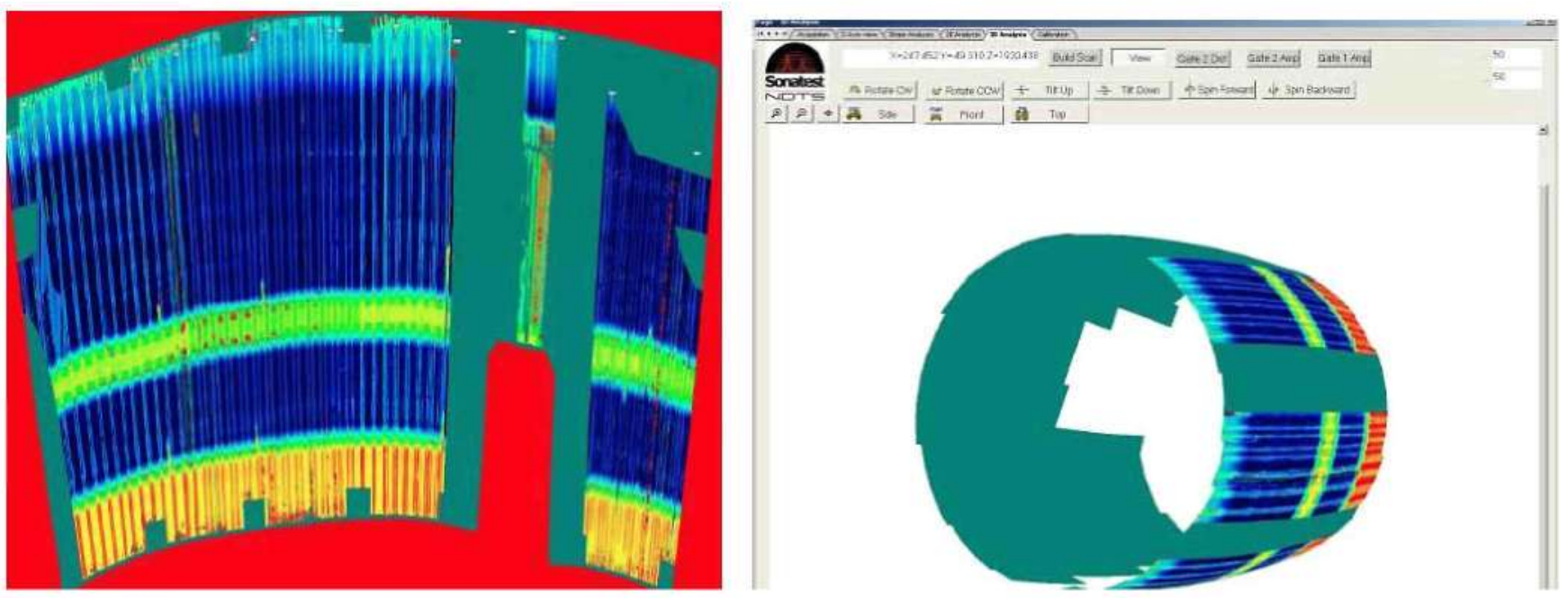

Fig. 7. Generated C-Scan inspection file. 2D (left) representation and 3D representation (right). 


\section{CONCLUSION}

The inspection system has shown good performance in the inspection of the extemal surface of the fuselage of an aircraft. The basic elements in its functioning are the generation of inspection trajectories that guarantee complete coverage of the part's snrface, the system for correcting the robot's trajectory in real time and the ultrasound sensor that captures the data corresponding to the inspected surface.

Trajectories have been generated according to the behavior of the trajectory correction systems, in such a way that in the areas of constant curvature the inspection Irajectories have followed the axis of the part, while in double curvature areas, the robot movement is executed according to the line of maximum slope.

The trajectory correction system is based on the use of four laser sensors that give a very precise measure of the distance of the sensors from the surface of the part. These sensors have been found to perform very well in maintaining the distance between the ultrasound sensors and the part, and also in the correction of the roll and pitch angles. It ensures that the contact of the ultrasonnd dnring the inspection is done. Finally, the software used in the processing of the data of the ultrasound echoes, along with the comesponding spatial positions provided by the robot, have enabled a 3D reconstrnction of the inspected part to be created.

The integration of the aforementioned modnles has been possible thanks to the collaboration of a transnational and multifunctional team, which has permitted the automatization of the inspection of the external surface of the sections of an aircraft fnselage using the technology known as "contact phased-aray wheel-probe".

\section{ACKNOWLEDGMENT}

This project and its development were made possible by its partners and the many other people who have worked on it. Suppliers such as KUKA. NDTS and also the incalculable contribntion of professionals from the School of Industrial Engineering at the Universidad Politécnica de Madrid. Last, but not least. we would like to thank the persounel from different areas (Engineering. Materials \& Processes. Development Technologies, and Antomation Technologies) of Airbus Spain who have been working hard for the snccess of this project.

\section{REFERENCES}

[1] [1] S. E. Black, R. L. Goodnan, K. N. Wood, "Multi-Channel DeepMennory Digitizing Architecture for Automated Inspection of Large Composite Surfaces". IEEE Systems Readiness Teclnnology Conference, California (USA), 18-21 Sept. 2006. pp. $558-564$.

[2] A. Maurer, W. Haase, R. Hetrich, R. Koch, W. de Odorico, "Automated Inspection of Conplex Composite Components in the
Production Cycle". Proc. of the 17th World Conference on Nondestructive Testing, Shanghai (China), Oct. 2008, pp: 25-28.

[3] D. Roach, "Assessing conventional and advanced NDI for composite Aircrafl". High-Performance Composites Article Archive. Available: http: iww compositesworld.con/articles/assessing-conventionalandadvanced-ndi-for-composite-aircraft.aspx), July 2008.

[4] Exper Poogranming KUKA Systen Soltware Release 4.1. 2005.

[5] Function Generator System Tech. KUKA System Soltware Release 5.1. 2005.

[6] A. Blomdell et al, "Extending an Industrial Robot Controller. Inplementation and Applications of a Fast Open Sensor Interface". IEEE Robotics \& Autonation Magazine, vol. 12, o 3, pp: 85-94, Sept 2005.

[7] W. Sheng, N. Xi, M. Song and Y. Chen, "CAD-Guided Sensor Planning for Dimensional Inspection in Autonotive Manufacturing", IEEE/ASME Transactions on Mexhatronics, vol. 8, n. 3, pp: 372-380. Siept. 2003. 